\title{
Comparison of sinonasal quality of life and health status in patients undergoing microscopic and endoscopic transsphenoidal surgery for pituitary lesions: a prospective cohort study
}

\author{
Andrew S. Little, MD, ${ }^{1}$ Daniel F. Kelly, MD, ${ }^{2}$ John Milligan, MD, ${ }^{1}$ Chester Griffiths, MD, ${ }^{2}$ \\ Daniel M. Prevedello, MD, ${ }^{3}$ Ricardo L. Carrau, MD, ${ }^{3}$ Gail Rosseau, MD, ${ }^{4}$ Garni Barkhoudarian, MD, ${ }^{2}$ \\ Heidi Jahnke, RN, MSN, ${ }^{1}$ Charlene Chaloner, RN, ${ }^{2}$ Kathryn L. Jelinek, BS, ${ }^{3}$ Kristina Chapple, PhD, ${ }^{1}$ \\ and William L. White, MD' \\ 1Division of Neurological Surgery, Barrow Neurological Institute, St. Joseph's Hospital and Medical Center, Phoenix, Arizona; \\ 2Brain Tumor Center, John Wayne Cancer Institute, Santa Monica, California; ${ }^{3}$ Department of Neurosurgery, Ohio State \\ University, Columbus, Ohio; and ${ }^{4}$ Department of Neurosurgery, NorthShore University HealthSystem, Evanston, Illinois
}

OBJECT Despite the widespread adoption of endoscopic transsphenoidal surgery for pituitary adenomas, the sinonasal quality of life (QOL) and health status in patients who have undergone this technique have not been compared with these findings in patients who have undergone the traditional direct uninostril microsurgical technique. In this study, the authors compared the sinonasal QOL and patient-reported health status after use of these 2 surgical techniques.

METHODS The study design was a nonblinded prospective cohort study. Adult patients with sellar pathology and planned transsphenoidal surgery were screened at 4 pituitary centers in the US between October 2011 and August 2013. The primary end point of the study was postoperative patient-reported sinonasal QOL as measured by the Anterior Skull Base Nasal Inventory-12 (ASK Nasal-12). Supplementary end points included patient-reported health status estimated by the 8-Item Short Form Health Survey (SF-8) and EuroQol (EQ)-5D-5L instruments, and sinonasal complications. Patients were followed for 6 months after surgery.

RESULTS A total of 301 patients were screened and 235 were enrolled in the study. Of these, 218 were analyzed (111 microsurgery patients, 107 endoscopic surgery patients). Demographic and tumor characteristics were similar between groups ( $p \geq 0.12$ for all comparisons). The most common complication in both groups was sinusitis ( $7 \%$ in the microsurgery group, $13 \%$ in the endoscopic surgery group; $p=0.15$ ). Patients treated with the endoscopic technique were more likely to have postoperative nasal debridements $(p<0.001)$. The ASK Nasal-12 and SF-8 scores worsened substantially for both groups at 2 weeks after surgery, but then returned to baseline at 3 months. At 3 months after surgery, patients treated with endoscopy reported statistically better sinonasal QOL compared with patients treated using the microscopic technique $(p=0.02)$, but there were no significant differences at any of the other postoperative time points.

CONCLUSIONS This is the first multicenter study to examine the effect of the transsphenoidal surgical technique on sinonasal QOL and health status. The study showed that surgical technique did not significantly impact these patientreported measures when performed at high-volume centers.

Clinical trial registration no.: NCT01504399 (clinicaltrials.gov).

http://thejns.org/doi/abs/10.3171/2014.10.JNS14921

KEY WORDS quality of life; endoscopic surgery; transsphenoidal surgery; pituitary adenoma; sinusitis; pituitary surgery 
$\mathrm{E}$ NDONASAL transsphenoidal surgery represents the treatment of choice for most symptomatic pituitary lesions. Because this approach exploits the nasal corridor and paranasal sinuses to reach the skull base, nasal trauma is a primary source of postoperative morbidity in many of these patients. Reported rhinological complications include sinusitis, synechiae, anosmia, epistaxis, septal perforation, nasal congestion, and impaired mucociliary clearance; these complications can adversely affect patients' nasal functioning, satisfaction with surgery, and quality of life (QOL). 1,4,5,13,16,33,35

Microscopic transsphenoidal surgery is the current gold standard for treating pituitary lesions. Although many variations in technique are practiced, transsphenoidal surgery often involves a uninostril exposure maintained by a nasal speculum, with visualization provided by an operating microscope..$^{10,14,15,28,34}$ Recently, some pituitary surgeons have adopted an endoscopic transsphenoidal technique that commonly uses a binostril exposure and an endoscope to provide panoramic visualization without a nasal speculum.9.19-21 In microscopic surgery, the surgeon expands the nasal corridor with a speculum, which stretches the nasal vestibule and fractures the turbinates. In contrast, in the endoscopic technique, the surgeon creates the surgical corridor by removing nasal tissue (for example, by creating a posterior septectomy or posterior ethmoidectomy) to accommodate the endoscope and dissectors.

Despite the widespread adoption of endoscopic transsphenoidal surgery for sellar pathology and other more extensive skull base lesions, the sinonasal morbidity and QOL of patients undergoing this approach have not been compared with those undergoing traditional, direct microsurgical techniques. In this prospective cohort study, we compare sinonasal morbidity, patient-reported QOL, and health status in patients who have undergone direct microscopic transsphenoidal surgery with these findings in patients who have undergone endoscopic transsphenoidal surgery for pituitary lesions.

\section{Methods \\ Study Design}

The study design was a nonblinded exploratory prospective cohort study comparing 2 commonly used transsphenoidal surgery techniques (i.e., direct uninostril endonasal microscopic approach and binostril endoscopic endonasal approach). The prespecified target enrollment was 200 patients, with 100 patients per cohort-200 participants provided $90 \%$ power to detect an effect size of 0.20 between the 2 surgical groups with an alpha set at 0.05 . This study was approved by the institutional review boards at the 4 participating institutions and was registered with the ClinicalTrials.gov database (http://clinicaltrials.gov); its registration no. is NCT01504399. Informed consent was obtained from all study participants. The study adhered to the principles set forth in the US Code of Federal Regulations, Title 45, Part 46, "Protection of Human Subjects" (revised January 15, 2009).

\section{Patient Population}

Eligible patients were consecutive English- or Spanish- speaking adults (18 years or older) with sellar pathology who underwent a planned primary or secondary transsphenoidal surgery in the outpatient neurosurgical clinics at 4 US pituitary centers between October 2011 and August 2013. Patients with a history of prior radiotherapy to the paranasal sinus region or skull base were excluded. A total of 301 patients were screened for eligibility; 47 patients were not eligible for the study and 19 declined to participate (Fig. 1). From this population, 235 patients received surgical treatment under the protocol.

\section{Study End Points}

The prespecified primary end point of the study was patient-reported nasal QOL, which was determined using the Anterior Skull Base Nasal Inventory-12 (ASK Nasal-12) at 2 weeks, 3 months, and 6 months after surgery. ${ }^{23}$ Secondary supplementary end points included health status estimated by patient scores on the 8-Item Short Form Health Survey (SF-8). ${ }^{7,32}$ The EuroQol (EQ)-5D-5L was also administered to patients at the lead site. ${ }^{17}$

Major surgical complications were also tracked. Syndrome of inappropriate antidiuretic hormone was defined as a hospital readmission for hyponatremia. A CSF leak was defined as a postoperative leak requiring surgical repair or lumbar drainage. Permanent diabetes insipidus was defined as new postoperative diabetes insipidus still present at the 6-month follow-up visit. Sinusitis was defined as facial pressure, nasal drainage, and the initiation of antibiotics. Epistaxis was defined as postoperative nosebleeds requiring an intervention such as nasal packing, cautery, or angiography. Other major surgical complications were also tracked, such as thromboembolic events detected on ultrasonography or CT angiography.

\section{Data Collection and Analysis}

Study data, including patient demographics, surgical approach details, and patient-reported end points, were collected and entered into a research database template by a designated research nurse at each participating site. The QOL instruments were self-administered in outpatient clinics. De-identified patient information was then forwarded to the lead site at the conclusion of the study. The SF-8 responses were scored using the scoring tool provided by Quality Metric, and the EQ-5D-5L responses were scored as described in the EuroQol Group user manual. Data were independently analyzed by a biostatistician affiliated with the lead institution.

\section{Surgical Approach and Postoperative Nasal Care}

The surgical approach for each patient was chosen by the treating neurosurgeon. Surgical technique details, such as treatment of the middle turbinate, sellar repair technique, and nasal packing, were tracked. To allow the study of common clinical practices, there was no prescribed postoperative nasal care protocol. Nasal rinses and endoscopic debridements were prescribed at the discretion of the treating team.

\section{Statistical Analysis}

Pearson correlations were used to analyze the asso- 


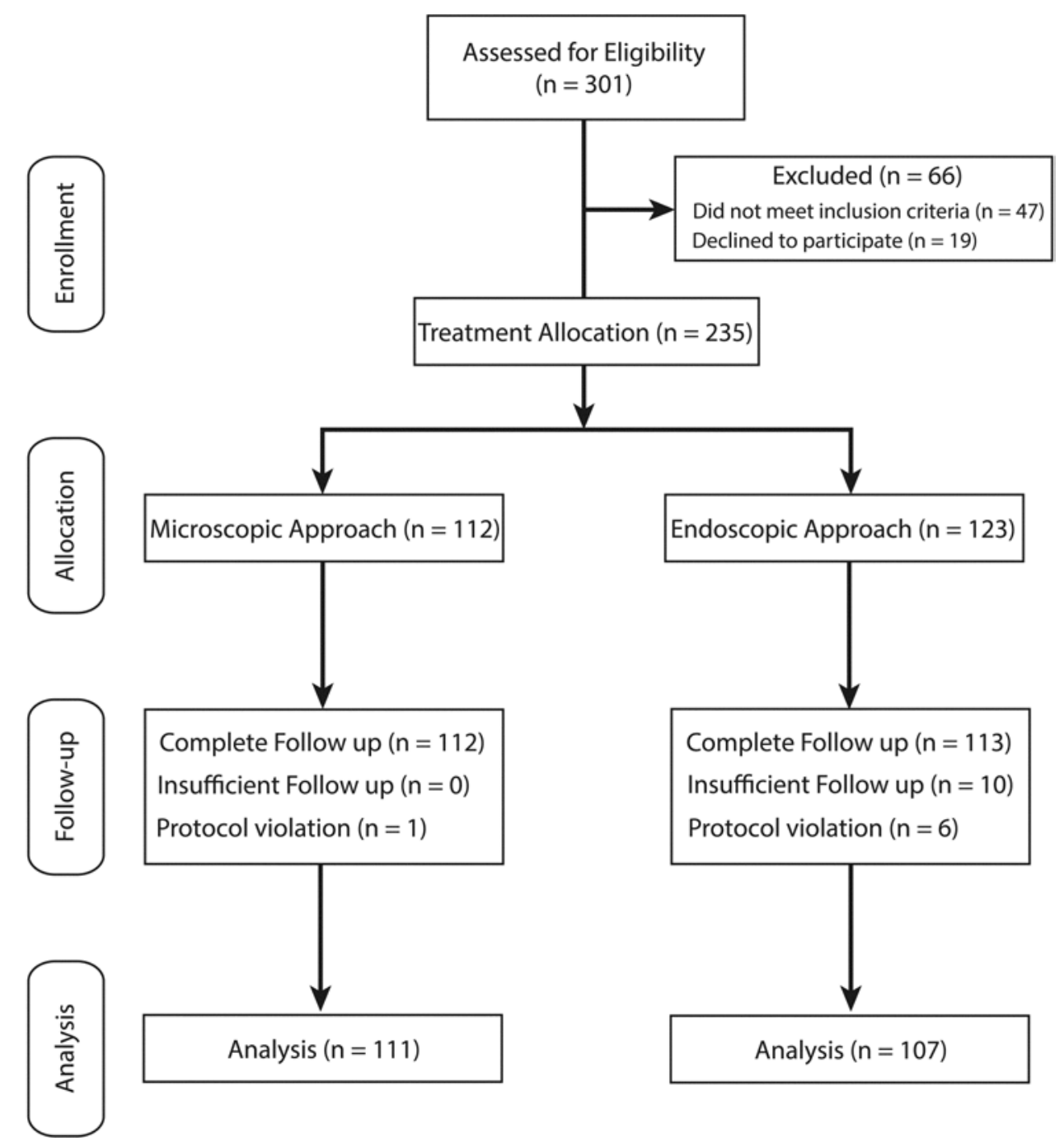

FIG. 1. The CONSORT flow diagram for this study.

ciation between the ASK Nasal-12, SF-8, and EQ-5D-5L visual analog scales and index scales. Differences in outcome variables by surgical technique were tested using an independent samples t-test for continuous variables and chi-square for ordinal variables. Repeated-measures general linear models were used to assess differences in the ASK Nasal-12, SF-8, and EQ-5D-5L visual analog scales and index scales over time and according to which surgical technique was used. The institution at which surgery was performed was included in our models to account for potential differences in patient self-reports by site. In cases where the assumption of sphericity was not met, as indicated by Mauchly's test, the Greenhouse-Geisser correction was applied. Statistical software (SPSS version 21) was used for all analyses. The $p$ values presented are 2 -tailed and considered significant at $\mathrm{p}<0.05$.

\section{Results}

Four pituitary centers screened patients for eligibility and 3 centers successfully enrolled patients. Three par- ticipating surgeons practiced endoscopic surgery and 2 practiced microscopic surgery. In total, 301 patients were screened and 235 patients underwent surgical treatment under the protocol. Enrollment was halted because the prespecified number of subjects had been enrolled. Ten patients' records were removed from the analysis because of insufficient follow-up and 7 were removed because the individuals underwent extended transsphenoidal approaches (a study exclusion criterion), leaving 218 patients in the final analysis. Of the 218 patients included, 111 were treated in the microscopic and 107 in the endoscopic surgery group. The groups were analyzed using an "as-treated" analysis. The Consolidated Standards for Reporting of Trials (CONSORT) flow diagram is presented in Fig. 1 . The groups were well matched with respect to baseline characteristics and demographics, including age, sex, tumor type and size, and history of prior sinus or transsphenoidal surgery ( $p \geq 0.12$ for all comparisons; Table 1$)$.

Table 2 describes technical details of the surgical approaches. The table highlights the differences in how the surgical techniques are commonly practiced. For example, 
TABLE 1. Demographic characteristics in 218 patients with pituitary lesions

\begin{tabular}{lccc}
\hline \multicolumn{1}{c}{ Characteristic } & $\begin{array}{c}\text { Microscopic } \\
\text { Op }\end{array}$ & $\begin{array}{c}\text { Endoscopic } \\
\text { Op }\end{array}$ & p Value \\
\hline No. of patients & 111 & 107 & \\
\hline Age in yrs, mean \pm SD & $51.8 \pm 17.1$ & $52.1 \pm 15.4$ & 0.88 \\
\hline Male patients & $55(50 \%)$ & $49(46 \%)$ & 0.58 \\
\hline Tumor type & & & 0.12 \\
\hline Nonfunctioning & $62(56 \%)$ & $72(67 \%)$ & \\
\hline Acromegaly & $15(14 \%)$ & $9(8 \%)$ & \\
\hline Cushing's & $10(9 \%)$ & $7(7 \%)$ & \\
\hline Prolactinoma & $11(10 \%)$ & $15(14 \%)$ & \\
\hline Rathke's cleft cyst & $6(5 \%)$ & $3(3 \%)$ & \\
\hline Others* & $7(6 \%)$ & $1(1 \%)$ & \\
\hline Tumor size in mm, mean \pm SD & $18.6 \pm 9.5$ & $18.1 \pm 10.3$ & 0.71 \\
\hline Prior TS surgery & $10(9 \%)$ & $10(9 \%)$ & 0.93 \\
\hline History of sinusitis & $12(11 \%)$ & $13(12 \%)$ & 0.76 \\
\hline Prior sinus surgery & $8(7 \%)$ & $7(7 \%)$ & 0.85 \\
\hline TS = transsphenoidal. & & \\
* Category includes pituitary cyst, metastasis, craniopharyngioma, abscess.
\end{tabular}

in the use of the binostril technique, an otolaryngology cosurgeon, nasal splints, absorbable nasal packing, and dural sealant were more common in the endoscopic group $(\mathrm{p} \leq$ 0.03). In contrast, the use of autologous fat graft, Medpore (Stryker) sellar reconstruction, nonabsorbable nasal packing, and the uninostril technique were more common in the microscopic group ( $\mathrm{p} \leq 0.01$ for all comparisons).

Postoperative nasal care was also examined (Table 3). Endoscopic patients were more likely to undergo nasal debridement in an otolaryngologist's office (76\% compared with $6 \%$ for microscopically treated patients, $\mathrm{p}<0.001$ ). The use of saltwater nasal irrigation was the most common nasal care technique in both groups.

There were no significant differences between groups in postoperative complications (Table 4). The most common complications in both groups were sinusitis requiring antibiotic treatment (7\% microscopic, 13\% endoscopic; $\mathrm{p}=0.15)$ and readmission for hyponatremia (4\% microscopic, $5 \%$ endoscopic; $\mathrm{p}=0.75)$. There was 1 death $(1 \%)$ due to intracerebral hemorrhage in the microscopic group. Postoperative CSF leak requiring intervention occurred in 2 patients $(2 \%)$ in the microscopic group and 1 patient (1\%) in the endoscopic group.

Demographic information and outcome measures were compared by site to justify including data from multiple sites into a single analysis. There were no significant differences by site for the following demographic data: patient age, sex, prior pituitary surgery, history of sinus surgery, history of chronic sinusitis, or tumor size. The ASK Nasal-12, SF-8, and EQ-5D-5L scores were also compared at each time point by site. Scores for ASK Nasal-12 at 2 weeks and 3 months and for SF-8 at 3 months were significantly different by site, although the effect sizes for all 3 significant comparisons were $\leq 0.03$, indicating a small effect. Therefore, site was included as a covariate in the repeated-measures models to account for variation
TABLE 2. Characteristics of the surgical technique performed in 218 patients with pituitary lesions

\begin{tabular}{|c|c|c|c|}
\hline Characteristic & $\begin{array}{c}\text { Microscopic } \\
\text { Op }\end{array}$ & $\begin{array}{c}\text { Endoscopic } \\
\text { Op }\end{array}$ & p Value \\
\hline No. of patients & 111 & 107 & \\
\hline Nostril & & & $<0.001$ \\
\hline Uninostril & $107(96 \%)$ & $4(4 \%)$ & \\
\hline Binostril & $4(4 \%)$ & $103(96 \%)$ & \\
\hline Nasal splints & & & $<0.001$ \\
\hline Yes & $1(1 \%)$ & $15(14 \%)$ & \\
\hline No & $110(99 \%)$ & $92(86 \%)$ & \\
\hline Septal mucosal flap & & & 0.09 \\
\hline Yes & $1(1 \%)$ & $5(5 \%)$ & \\
\hline No & $110(99 \%)$ & $102(95 \%)$ & \\
\hline Autologous fat graft & & & 0.01 \\
\hline Yes & $80(72 \%)$ & $60(56 \%)$ & \\
\hline No & $31(28 \%)$ & $47(44 \%)$ & \\
\hline Dural sealant & & & 0.03 \\
\hline Yes & $32(29 \%)$ & $46(43 \%)$ & \\
\hline No & $79(71 \%)$ & $61(57 \%)$ & \\
\hline Sellar implant & & & $<0.001$ \\
\hline Absorbable plate & $0(0 \%)$ & $6(6 \%)$ & \\
\hline Medpore & $75(67 \%)$ & $41(38 \%)$ & \\
\hline Titanium & $0(0 \%)$ & $3(3 \%)$ & \\
\hline Allograft & $0(0 \%)$ & $1(1 \%)$ & \\
\hline Septal autograft & $13(12 \%)$ & $10(9 \%)$ & \\
\hline None & $23(21 \%)$ & $46(43 \%)$ & \\
\hline Nonabsorbable nasal packing & & & $<0.001$ \\
\hline Yes & $109(98 \%)$ & $9(8 \%)$ & \\
\hline No & $2(2 \%)$ & $98(92 \%)$ & \\
\hline Absorbable nasal packing & & & $<0.001$ \\
\hline Yes & $5(5 \%)$ & $24(22 \%)$ & \\
\hline No & $106(96 \%)$ & $83(78 \%)$ & \\
\hline Otolaryngology co-surgeon & & & $<0.001$ \\
\hline Yes & $1(1 \%)$ & $58(54 \%)$ & \\
\hline No & $110(99 \%)$ & $49(46 \%)$ & \\
\hline Middle turbinate & & & 0.24 \\
\hline Out-fracture & $111(100 \%)$ & $105(98 \%)$ & \\
\hline In-fracture & $0(0 \%)$ & $2(2 \%)$ & \\
\hline Resect & $0(0 \%)$ & $0(0 \%)$ & \\
\hline
\end{tabular}

between sites. We also separately examined the influence of surgeon experience on sinonasal QOL, and we found that surgeon experience was not correlated with sinonasal QOL at any time point.

\section{Sinonasal QOL and Health Status}

The primary end point of the study was patient-reported nasal QOL as measured by the ASK Nasal-12. Patients in both the microscopic and endoscopic groups reported a marked worsening of sinonasal symptoms at 2 weeks after surgery; however, symptoms returned to baseline at 3 months (Table 5). Univariate comparisons of sinonasal 
TABLE 3. Postoperative nasal care in 218 patients with pituitary lesions

\begin{tabular}{lccc}
\hline \multicolumn{1}{c}{ Characteristic } & $\begin{array}{c}\text { Microscopic } \\
\text { Op }\end{array}$ & $\begin{array}{c}\text { Endoscopic } \\
\text { Op }\end{array}$ & p Value \\
\hline No. of patients & 111 & 107 & \\
\hline Nasal care & & & 0.01 \\
\hline Saltwater spray & $110(99 \%)$ & $93(87 \%)$ & \\
\hline Neti pot & $1(1 \%)$ & $5(5 \%)$ & \\
\hline None & $0(0 \%)$ & $9(8 \%)$ & \\
\hline Nasal debridements after op & & & $<0.001$ \\
\hline 0 & $105(94 \%)$ & $26(24 \%)$ & \\
\hline 1 & $3(3 \%)$ & $28(26 \%)$ & \\
\hline 2 & $3(3 \%)$ & $12(11 \%)$ & \\
\hline 3 & $0(0 \%)$ & $15(14 \%)$ & \\
\hline 4 or more & $0(0 \%)$ & $26(24 \%)$ & \\
\hline
\end{tabular}

symptoms between patients who received microscopic and endoscopic surgery revealed that there were no significant differences in overall mean ASK Nasal-12 scores preoperatively, at 2 weeks, or at 6 months (Fig. 2, Table 5). However, there was a significant difference in mean scores at 3 months, with those in the endoscopic group having less severe symptoms (microscopic 0.99 [95\% CI 0.83-1.16], endoscopic 0.72 [95\% CI 0.55-0.89]; $p=0.02$ ); however, the effect size was small, with a partial eta-squared value of 0.37 . The repeated-measures models revealed a cubic "s"-shaped effect for time $\left(\mathrm{F}_{1,189}=51.7, \mathrm{p}<0.001\right)$ that is visible on the box plots, with scores increasing at 2 weeks, sharply decreasing at 3 months, and slightly decreasing at 6 months (Fig. 2A). The group effect of surgical approach was not significant in this or any of the other repeatedmeasures models.

The univariate analyses and repeated-measure models described above for the ASK Nasal-12 were also used to describe trends in patient-reported SF-8 scores, EQ-5D$5 \mathrm{~L}$ visual analog scale scores, and EQ-5D-5L index scores over time. As shown in Table 5, there were no statistically significant differences by surgical technique at any postoperative time point for the SF-8 or the EQ-5D-5L visual analog scale; however, preoperative EQ-5D-5L index scores were more severe for the endoscopic group $(\mathrm{p}=$ $0.02)$. Similar to the ASK Nasal-12, a cubic effect for time emerged for the SF-8 $\left(\mathrm{F}_{1.189}=51.5, \mathrm{p}<0.001\right)$, and a linear time effect for the EQ-5D-5L visual analog scale was noted $\left(\mathrm{F}_{1,51}=12.1, \mathrm{p}<0.001\right)$ (Fig. $2 \mathrm{~B}$ and $\left.\mathrm{C}\right)$.

An item-level analysis of ASK Nasal-12 was performed to determine whether there were differences in individual symptoms. At 3 months, "whistling sound" ( $p=0.01)$, "sense of taste" ( $p=0.03)$, and "sense of smell" $(p=0.01)$ were significantly better in the endoscopic compared with the microscopic surgery group. There were no significant differences at 2 weeks or 6 months.

\section{Discussion}

The QOL and other patient-reported end points are important clinical metrics to help supplement objective surgical outcomes. These end points are becoming in-
TABLE 4. Postoperative complications by treatment group in 218 patients with pituitary lesions

\begin{tabular}{lccc}
\hline \multicolumn{1}{c}{ Complication* } & $\begin{array}{c}\text { Microscopic } \\
\text { Op }\end{array}$ & $\begin{array}{c}\text { Endoscopic } \\
\text { Op }\end{array}$ & p Value \\
\hline No. of patients & 111 & 107 & \\
\hline Visual decline & $2(2 \%)$ & 0 & 0.16 \\
\hline SIADH & $4(4 \%)$ & $5(5 \%)$ & 0.75 \\
\hline CSF leak & $2(2 \%)$ & $1(1 \%)$ & 0.58 \\
\hline Sinusitis & $8(7 \%)$ & $14(13 \%)$ & 0.15 \\
\hline Epistaxis & $2(2 \%)$ & $0(0 \%)$ & 0.16 \\
\hline Intracranial hematoma & $2(2 \%)$ & $0(0 \%)$ & 0.16 \\
\hline Permanent DI & $4(4 \%)$ & $2(2 \%)$ & 0.45 \\
\hline Thromboembolism & $1(1 \%)$ & $1(1 \%)$ & 1.0 \\
\hline Meningitis & 0 & 0 & 1.0 \\
\hline Carotid artery injury & 0 & 0 & 1.0 \\
\hline Death & $1(1 \%)$ & 0 & 1.0 \\
\hline
\end{tabular}

$\mathrm{DI}=$ diabetes insipidus; SIADH = syndrome of inappropriate antidiuretic hormone.

* Complications were defined as indicated in the Methods.

creasingly reported in endonasal skull base surgical series because they represent patient-reported impressions and provide valuable insight for practitioners. Sinonasal complaints are the primary postoperative morbidity in most patients undergoing elective transsphenoidal surgery for pituitary lesions. In this study, we sought to compare patient-reported nasal morbidity and QOL for the 2 most common transsphenoidal surgical techniques (microscopic direct uninostril with nasal speculum and endoscopic binostril technique).

We learned that, overall, sinonasal QOL and objective sinonasal complications such as sinusitis were similar in the 2 groups in the 6 months after surgery. Patient nasal QOL scores favored endoscopic surgery at 3 months, but the effect size was small and there was no difference at 6 months, suggesting that the difference seen at 3 months was not clinically significant. Sinonasal symptoms worsened in both groups in the immediate postoperative period and then returned to baseline by 3 months after surgery, demonstrating a cubic s-shaped time effect. As supplementary end points, we also assessed overall health status as determined by patient responses on SF-8 and EQ-5D$5 \mathrm{~L}$ instruments. These data suggest that these patient-reported QOL measures are not affected by the differences between microscopic and endoscopic surgical approaches and postoperative care paradigms when performed in the setting of established pituitary programs.

Previous studies have demonstrated that sinonasal symptoms such as nasal congestion and discharge worsen significantly in the perioperative period, but improve to near baseline by 3-6 months after surgery. $2,8,24,25,27,31$ Factors associated with increased nasal symptoms after surgery include the use of an extended endonasal approach, septal-mucosal graft, and residual tumor after surgery. ${ }^{3}$ The strengths of these studies include the use of validated outcome measures and longitudinal follow-up that allows factors that influence QOL to be meaningfully analyzed..$^{11,12,18,29}$ The primary limitations of these studies 
TABLE 5. Responses on 3 QOL instruments by treatment group in 218 patients with pituitary lesions

\begin{tabular}{|c|c|c|c|c|}
\hline \multirow[b]{2}{*}{ Instrument } & \multicolumn{2}{|c|}{ Mean Score (SE) $[95 \% \mathrm{Cl}]$} & \multicolumn{2}{|c|}{$p$ Value } \\
\hline & Microscopic Op & Endoscopic Op & $\begin{array}{l}\text { Op Techs Compared } \\
\text { at Each Time Point }\end{array}$ & $\begin{array}{c}\left.\text { Time (partial } \eta^{2}\right) \\
\text { [trend] }\end{array}$ \\
\hline ASK Nasal-12* & & & & $<0.001(0.37)$ [cubic] \\
\hline Preop & $0.82(0.08)[0.67-0.97]$ & $0.73(0.08)[0.58-0.89]$ & 0.67 & \\
\hline 2 wks & $1.78(0.09)$ [1.59-1.96] & $1.62(0.10)$ [1.43-1.81] & 0.23 & \\
\hline $3 \mathrm{mos}$ & $0.99(0.08)$ [0.83-1.16] & $0.72(0.09)$ [0.55-0.89] & 0.02 & \\
\hline $6 \mathrm{mos}$ & $0.73(0.08)[0.56-0.89]$ & $0.76(0.09)[0.59-0.93]$ & 0.54 & \\
\hline SF-8† & & & & $<0.001(0.22)$ [cubic] \\
\hline Preop & $45.25(1.18)[42.93-47.56]$ & $45.02(1.19)[42.67-47.37]$ & 0.45 & \\
\hline 2 wks & $39.82(1.05)$ [37.74-41.90] & $39.23(1.07)[37.12-41.34]$ & 0.38 & \\
\hline $3 \mathrm{mos}$ & $46.50(1.06)$ [44.41-48.58] & $47.64(1.07)$ [45.53-49.75] & 0.81 & \\
\hline $6 \mathrm{mos}$ & $47.97(1.10)$ [45.79-50.15] & $48.97(1.12)$ [46.76-51.18] & 0.73 & \\
\hline \multicolumn{5}{|l|}{$E Q-5 D-5 L$} \\
\hline Visual analog scale $†$ & & & & $<0.001(0.24)$ [linear] \\
\hline Preop & 73.39 (3.96) [65.43-81.35] & $60.25(4.93)[50.34-70.16]$ & 0.08 & \\
\hline 2 wks & $71.77(3.43)$ [64.87-78.68] & $67.00(4.28)[58.40-75.60]$ & 0.92 & \\
\hline $3 \mathrm{mos}$ & 80.61 (3.34) [73.90-87.33] & $73.00(4.16)$ [64.64-81.36] & 0.18 & \\
\hline $6 \mathrm{mos}$ & $82.10(3.89)$ [74.27-89.92] & $73.05(4.85)$ [63.31-82.79] & 0.11 & \\
\hline Index score $†$ & & & & $0.19(0.03)[\mathrm{NA}]$ \\
\hline Preop & $0.82(0.03)[0.76-0.88]$ & $0.73(0.04)[0.66-0.80]$ & 0.02 & \\
\hline 2 wks & $0.79(0.02)[0.74-0.84]$ & $0.76(0.03)[0.70-0.82]$ & 0.74 & \\
\hline $3 \mathrm{mos}$ & $0.82(0.03)[0.76-0.89]$ & $0.78(0.04)[0.69-0.86]$ & 0.29 & \\
\hline $6 \mathrm{mos}$ & $0.82(0.03)[0.76-0.88]$ & $0.80(0.04)[0.72-0.88]$ & 0.47 & \\
\hline
\end{tabular}

are that they report on single-institution experiences and a diverse set of skull base pathologies. Our study builds on this prior work because it reports on a multicenter prospective experience, uses validated outcome measures, studies a large sample size, and examines a narrower patient population limited to sellar pathology that allows for more specific comparisons. Finally, this current study examines the impact of surgical technique on QOL, which previous work has not fully investigated.

Because this study used a prospective pituitary surgery protocol for which data were collected by dedicated research nurses and adjudicated by physicians, it offers a level of data quality that exceeds previous national surveys of pituitary surgeons and reviews of administrative data sets. ${ }^{6,22}$ In addition, because of its multicenter design, the study also provides a current snapshot of microscopic and endoscopic transsphenoidal surgical practices and outcomes. For example, endoscopic surgeons in this study used middle turbinate out-fracture rather than turbinate resection to widen the endonasal corridor. The initial descriptions of endoscopic endonasal surgery proposed removing the middle turbinate, but we have found that to be unnecessary for most pituitary surgery. ${ }^{21}$ We also learned that vascularized septal mucoperiosteal flaps are not commonly used in routine endoscopic transsphenoidal surgery, because most CSF leaks encountered are low-flow fistulas and because of the increased nasal morbidity encountered using this technique. ${ }^{8,27}$ This study also provides a benchmark for postoperative complications in transsphenoidal surgery. The most common reason for readmission in the first 30 days after surgery was hyponatremia from syndrome of inappropriate antidiuretic hormone, which suggests a rational target for improving readmission rates. Thus, we subsequently developed an outpatient sodium screening protocol to prevent readmissions by identifying at-risk patients.

Several points about the study deserve further discussion. First, the goal of the study was to evaluate 2 commonly performed transsphenoidal surgical techniques and their typical postoperative nasal care regimens. For that reason, the study protocol did not prescribe specific postoperative nasal care techniques. Centers were encouraged to use routine nasal irrigation and "as needed" sinus debridement in symptomatic patients. However, routine postoperative nasal debridement was not required as part of the protocol, even though it is standard at some centers. Patients treated with endoscopic surgery were much more likely to have undergone a nasal debridement than the ones receiving microscopic surgery, reflecting the common differences in the postoperative care paradigms for these patients. Second, the study was designed as an observational protocol rather than a randomized study, reflecting the fact that a randomized study was impractical to conduct. Most centers have only 1 or 2 specialized transsphenoi- 

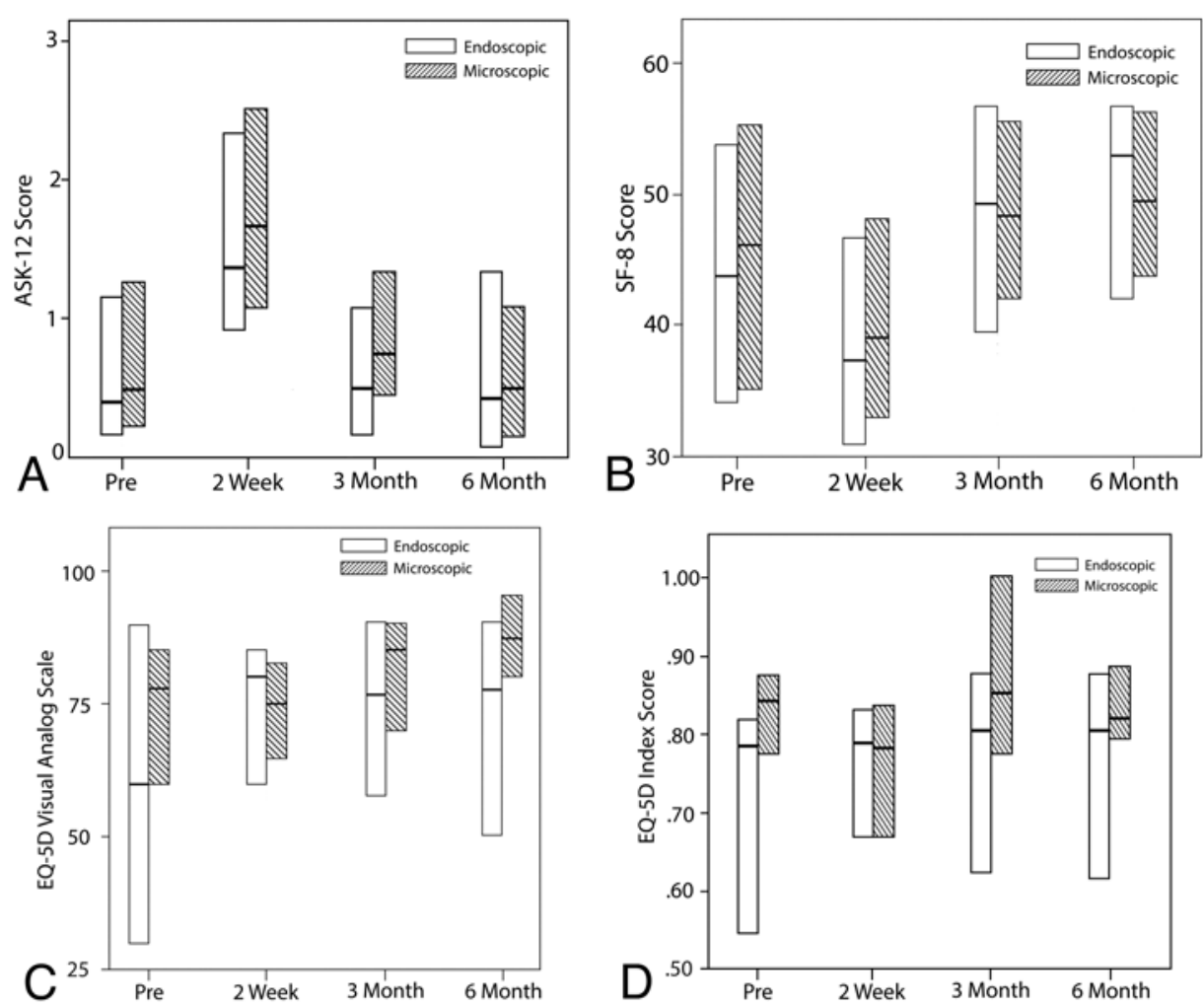

FIG. 2. Box plots of patient-reported (A) sinonasal QOL, (B) SF-8, (C) EQ-5D-5L visual analog scale, and (D) EQ-5D-5L index health status scores. Higher scores for sinonasal QOL indicate worse symptoms. Lower scores for SF-8 and EQ-5D-5L indicate worse health status. The middle line inside each box represents the median. Upper and lower horizontal lines on each box represent the 75th and 25th percentiles, respectively.

dal surgeons who practice either an endoscopic technique or a microscopic technique. Third, because of its observational nature, this study may contain certain biases. The most likely source is referral bias to pituitary centers. With respect to surgeon selection bias, participating surgeons did not select patients for surgical technique based on tumor characteristics. For example, patients with large lesions with suprasellar extension or cavernous sinus invasion, situations in which endoscopic surgery may have an advantage compared with microscopic surgery, were not preferentially selected for endoscopic surgery. ${ }^{9,26}$ As support for this statement, baseline tumor characteristics were well matched between the 2 groups. Fourth, with respect to trial generalizability, our study compared the direct uninostril microscopic technique with the binostril endoscopic technique and their typical postoperative nasal care regimens. Our results would not be expected to be applicable to the other major transsphenoidal surgical techniques, such as a uninostril endoscopic technique or a sublabial microscopic technique. Fifth, otolaryngology participation in surgery and follow-up differed by technique and neurosurgeon, reflecting contemporary practices. Examining the impact of otolaryngology participation, nasal debridement, and sinus rinses on sinonasal QOL is an important future direction. We are therefore planning a post hoc analysis of other patient and surgical factors that might predict sinonasal outcome.

Finally, the study design and limitations warrant addi- tional comment. Because the study goal was to compare patient-reported outcomes and sinonasal QOL, the key baseline and follow-up data are patient-reported outcomes rather than the objective surgical outcomes that readers are accustomed to seeing in pituitary case series. Because of its design as a QOL study, the protocol does not address some very intriguing questions of interest to pituitary surgeons. For example, this study does not investigate differences in biochemical remission rates between microscopic and endoscopic cohorts in patients with functioning pituitary adenoma. This study is unable to address that point because the specified follow-up period of 6 months, which is sufficient for sinonasal healing, is not sufficient for hormonally active tumors such as Cushing's disease or acromegaly, for which it can take longer than 6 months to determine surgical remission status. In addition, the study was underpowered to evaluate functioning tumors. Functioning tumors represented only $30 \%$ of the study cohort. Notably, others have addressed this question. Starke and colleagues ${ }^{30}$ performed a retrospective comparison between a surgeon using a microscopic technique and a surgeon using an endoscopic technique in patients with acromegaly. These authors discovered that there was no significant difference in remission rate. Tumor anatomical factors and preoperative growth hormone levels were more important predictors of outcome. The other important question of interest to pituitary surgeons that this study did not address was whether surgical technique influenced extent of tumor resection. 
One of us has previously demonstrated that the application of endoscopy to microscopic surgery does improve tumor detection rates and extent of resection. ${ }^{26}$ Because of the limitations mentioned above, this study does not address which surgical technique has overall superiority (i.e., endocrine outcomes, extent of resection, and QOL).

\section{Conclusions}

This prospective, observational study compared sinonasal QOL and health status in patients who had undergone transsphenoidal surgery for pituitary pathology using either a direct uninostril microscopic technique with a nasal speculum or an endoscopic binostril technique. The study found that patients in both groups had initial worsening of their sinonasal QOL and health status during the perioperative period, but QOL improved at later follow-up. There were no clinically significant differences between techniques in the patient-reported measures reported in this study. When performed by surgeons at high-volume centers, both surgical techniques yield similar sinonasal QOL and health status results for patients.

\section{Acknowledgments}

We acknowledge the Barrow Neurological Foundation for providing funding for the study, and the Neuroscience Publications office at Barrow Neurological Institute for assistance with manuscript preparation.

\section{References}

1. Alobid I, Enseñat J, Mariño-Sánchez F, de Notaris M, Centellas S, Mullol J, et al: Impairment of olfaction and mucociliary clearance after expanded endonasal approach using vascularized septal flap reconstruction for skull base tumors. Neurosurgery 72:540-546, 2013

2. Balaker AE, Bergsneider M, Martin NA, Wang MB: Evolution of sinonasal symptoms following endoscopic anterior skull base surgery. Skull Base 20:245-251, 2010

3. Bedrosian JC, McCoul ED, Raithatha R, Akselrod OA, Anand VK, Schwartz TH: A prospective study of postoperative symptoms in sinonasal quality-of-life following endoscopic skull-base surgery: dissociations based on specific symptoms. Int Forum Allergy Rhinol 3:664-669, 2013

4. Cappabianca P, Cavallo LM, Colao A, de Divitiis E: Surgical complications associated with the endoscopic endonasal transsphenoidal approach for pituitary adenomas. J Neurosurg 97:293-298, 2002

5. Charalampaki P, Ayyad A, Kockro RA, Perneczky A: Surgical complications after endoscopic transsphenoidal pituitary surgery. J Clin Neurosci 16:786-789, 2009

6. Ciric I, Ragin A, Baumgartner C, Pierce D: Complications of transsphenoidal surgery: results of a national survey, review of the literature, and personal experience. Neurosurgery 40:225-237, 1997

7. Cunillera O, Tresserras R, Rajmil L, Vilagut G, Brugulat $P$, Herdman M, et al: Discriminative capacity of the EQ-5D, SF-6D, and SF-12 as measures of health status in population health survey. Qual Life Res 19:853-864, 2010

8. de Almeida JR, Snyderman CH, Gardner PA, Carrau RL, Vescan AD: Nasal morbidity following endoscopic skull base surgery: a prospective cohort study. Head Neck 33:547-551, 2011

9. Dehdashti AR, Ganna A, Karabatsou K, Gentili F: Pure en- doscopic endonasal approach for pituitary adenomas: early surgical results in 200 patients and comparison with previous microsurgical series. Neurosurgery 62:1006-1017, 2008

10. Fatemi N, Dusick JR, de Paiva Neto MA, Kelly DF: The endonasal microscopic approach for pituitary adenomas and other parasellar tumors: a 10 -year experience. Neurosurgery 63 (4 Suppl 2):244-256, 2008

11. Gil Z, Abergel A, Spektor S, Cohen JT, Khafif A, Shabtai E, et al: Quality of life following surgery for anterior skull base tumors. Arch Otolaryngol Head Neck Surg 129:13031309,2003

12. Gil Z, Abergel A, Spektor S, Shabtai E, Khafif A, Fliss DM: Development of a cancer-specific anterior skull base qualityof-life questionnaire. J Neurosurg 100:813-819, 2004

13. Gondim JA, Almeida JP, Albuquerque LA, Schops M, Gomes E, Ferraz T, et al: Endoscopic endonasal approach for pituitary adenoma: surgical complications in 301 patients. Pituitary 14:174-183, 2011

14. Hardy J: Transsphenoidal hypophysectomy. J Neurosurg 34:582-594, 1971

15. Hardy J, Vezina JL: Transsphenoidal neurosurgery of intracranial neoplasm. Adv Neurol 15:261-273, 1976

16. Hart CK, Theodosopoulos PV, Zimmer LA: Olfactory changes after endoscopic pituitary tumor resection. Otolaryngol Head Neck Surg 142:95-97, 2010

17. Herdman M, Gudex C, Lloyd A, Janssen M, Kind P, Parkin $\mathrm{D}$, et al: Development and preliminary testing of the new five-level version of EQ-5D (EQ-5D-5L). Qual Life Res 20:1727-1736, 2011

18. Hopkins C, Gillett S, Slack R, Lund VJ, Browne JP: Psychometric validity of the 22-item Sinonasal Outcome Test. Clin Otolaryngol 34:447-454, 2009

19. Jho HD, Alfieri A: Endoscopic endonasal pituitary surgery: evolution of surgical technique and equipment in 150 operations. Minim Invasive Neurosurg 44:1-12, 2001

20. Jho HD, Carrau RL: Endoscopic endonasal transsphenoidal surgery: experience with 50 patients. J Neurosurg 87:44-51, 1997

21. Kassam A, Snyderman CH, Mintz A, Gardner P, Carrau RL: Expanded endonasal approach: the rostrocaudal axis. Part I. Crista galli to the sella turcica. Neurosurg Focus 19(1):E3, 2005

22. Little AS, Chapple K: Predictors of resource utilization in transsphenoidal surgery for Cushing disease. J Neurosurg 119:504-511, 2013

23. Little AS, Kelly D, Milligan J, Griffiths C, Rosseau G, Prevedello DM, et al: Prospective validation of a patient-reported nasal quality-of-life tool for endonasal skull base surgery: the Anterior Skull Base Nasal Inventory-12. J Neurosurg 119:1068-1074, 2013

24. McCoul ED, Anand VK, Bedrosian JC, Schwartz TH: Endoscopic skull base surgery and its impact on sinonasal-related quality of life. Int Forum Allergy Rhinol 2:174-181, 2012

25. McCoul ED, Anand VK, Schwartz TH: Improvements in site-specific quality of life 6 months after endoscopic anterior skull base surgery: a prospective study. J Neurosurg 117:498-506, 2012

26. McLaughlin N, Eisenberg AA, Cohan P, Chaloner CB, Kelly DF: Value of endoscopy for maximizing tumor removal in endonasal transsphenoidal pituitary adenoma surgery. J Neurosurg 118:613-620, 2013

27. Pant H, Bhatki AM, Snyderman CH, Vescan AD, Carrau RL, Gardner P, et al: Quality of life following endonasal skull base surgery. Skull Base 20:35-40, 2010

28. Patel SK, Husain Q, Eloy JA, Couldwell WT, Liu JK: Norman Dott, Gerard Guiot, and Jules Hardy: key players in the resurrection and preservation of transsphenoidal surgery. Neurosurg Focus 33(2):E6, 2012

29. Piccirillo JF, Merritt MG Jr, Richards ML: Psychometric and 
clinimetric validity of the 20-Item Sino-Nasal Outcome Test (SNOT-20). Otolaryngol Head Neck Surg 126:41-47, 2002

30. Starke RM, Raper DM, Payne SC, Vance ML, Oldfield EH, Jane JA Jr: Endoscopic vs microsurgical transsphenoidal surgery for acromegaly: outcomes in a concurrent series of patients using modern criteria for remission. J Clin Endocrinol Metab 98:3190-3198, 2013

31. Suberman TA, Zanation AM, Ewend MG, Senior BA, Ebert CS Jr: Sinonasal quality-of-life before and after endoscopic, endonasal, minimally invasive pituitary surgery. Int Forum Allergy Rhinol 1:161-166, 2011

32. Turner-Bowker DM, Bayliss MS, Ware JE Jr, Kosinski M: Usefulness of the SF-8 Health Survey for comparing the impact of migraine and other conditions. Qual Life Res 12:1003-1012, 2003

33. Urquhart AC, Bersalona FB, Ejercito VS, Holt JJ: Nasal septum after sublabial transseptal transsphenoidal pituitary surgery. Otolaryngol Head Neck Surg 115:64-69, 1996

34. Wilson CB, Rand RW, Grollmus JM, Heuser G, Levin S, Goldfield E, et al: Surgical experience with a microscopic transsphenoidal approach to pituitary tumors and non-neoplastic parasellar conditions. Calif Med 117:1-9, 1972

35. Zada G, Kelly DF, Cohan P, Wang C, Swerdloff R: Endonasal transsphenoidal approach for pituitary adenomas and other sellar lesions: an assessment of efficacy, safety, and patient impressions. J Neurosurg 98:350-358, 2003

\section{Author Contributions}

Conception and design: Little, Kelly, Milligan, Griffiths, Prevedello, Carrau, Rosseau, Barkhoudarian, White. Acquisition of data: Jahnke, Chaloner, Jelinek. Analysis and interpretation of data: Little, Kelly, Barkhoudarian, Chapple. Drafting the article: Chapple. Critically revising the article: Little, Kelly, Barkhoudarian, Chapple. Reviewed submitted version of manuscript: Little, Kelly, Barkhoudarian. Approved the final version of the manuscript on behalf of all authors: Little. Statistical analysis: Chapple. Administrative/technical/material support: Jahnke. Study supervision: Little, Kelly.

\section{Correspondence}

Andrew S. Little, c/o Neuroscience Publications, Barrow Neurological Institute, St. Joseph's Hospital and Medical Center, 350 W. Thomas Rd., Phoenix, AZ 85013. email: neuropub@ dignityhealth.org. 